\title{
Antipartum surveillance by scoring system for pregnancy outcome of cesarean section in a tertiary referral centre of Madhya Pradesh, India
}

\author{
Sapna B. Jain, Rashmi Tripathi*
}

Department of Obstetrics and Gynecology, J. K. Hospital and L. N. Medical College, Bhopal, Madhya Pradesh, India

Received: 01 August 2018

Accepted: 08 August 2018

*Correspondence:

Dr. Rashmi Tripathi,

E-mail: drrashmitripathi@ hotmail.com

Copyright: (C) the author(s), publisher and licensee Medip Academy. This is an open-access article distributed under the terms of the Creative Commons Attribution Non-Commercial License, which permits unrestricted non-commercial use, distribution, and reproduction in any medium, provided the original work is properly cited.

\begin{abstract}
Background: It is imperative to find out high risk pregnancy for providing apt care to the needy mother and fetus, so application of risk scoring system helps in pin pointing the at-risk patient and also gives a notion about the prognosis of fetal and maternal wellbeing. This study aims to evaluate the clinical application of risk scoring system in cesarean delivery for identification and management of risk pregnancies appropriately to improve perinatal and maternal morbidity and mortality.

Methods: LNMC Bhopal M.P. a tertiary referral centre with approximately 2100 delivery / year and cesarean rate of $33 \%$, serving primarily rural population. This prospective analytical study conducted from July 2017 till June 2018. Focused on the combination of the principal predictors of obstetric outcome taken from the previously published scoring system were applied on 120 cases of unplanned at risk caesarean section and summed up to identify riskpregnancy and its predictive value for maternal and perinatal outcome.

Results: It was determined by birth weight, APGAR score, birth asphyxia and NICU admission, Perinatal and maternal morbidity and mortality. Out of 120 caesarean cases $25 \%$ grouped in high risk, $58.3 \%$ low risk and $16.6 \%$ moderate group and their perinatal outcome was compared. $90 \%$ and $15 \%$ of the high risk and moderate group respectively had high risk neonates. In the high-risk group $2 \%$ had neonatal death, while no mortality in low risk cases. Maternal outcome was analysed by HDU admission 3\% mothers were admitted in HDU with eclampsia and multi organ failure with one maternal mortality due to HELLP syndrome other recovered well, while one patient left against medical advice due to high expenses.

Conclusions: Risk evaluation by simplified scoring system is an easy and economical way to identify high risk pregnancy to provide quick, comprehensive and quality health services to needy mother and neonate at right time, thus help in lowering the perinatal and maternal mortality and morbidity even at PHC level.
\end{abstract}

Keywords: Caesarian delivery HDU, HRP, High risk factors, Perinatal morbidity, Scoring system

\section{INTRODUCTION}

Out of total complications of pregnancy, incidence of high risk pregnancy is $7-8 \%$, with disproportionately high incidence of perinatal and maternal morbidity and mortality. Any pregnant women can develop life threatening complications with little or no advance warning. The complication of pregnancy and labour are essentially of two types - obstetrics complications like
$\mathrm{PPH}, \mathrm{PET} / \mathrm{E}$ etc. which require intensive obstetric care by specially trained providers and the other set of complications are multi organ involvement which require care of intensivist and super specialist such as nephrology, neurology, cardiology, pulmonologyetc. So, a high-risk pregnancy (HRP) is one in which the maternal environment or past reproductive performance presents a significant risk to fetal well-being, such as premature birth, small for date infant, full term with retarded growth 
or still births and early neonatal death. Identification of patients at risk of these complicated pregnancies with poor outcome is fundamental to antenatal care to improve reproductive outcome. ${ }^{1}$ There should be a simple, economical and handy system to identify high risk pregnancy specially in high delivery load primary health enter. This risk scoring system is a formalized method flof recognizing, documenting and cumulating antepartum, intrapartum and neonatal risk factors in order to predict complications for the mother, fetus and new born as well as easy and prompt shifting of patient if required. ${ }^{2}$

The level of development in a community is often judged by its perinatal mortality rate which not only reflects the socioeconomic status, educational level and cultural practices of the mother and directly reflects the quality of medical care provided at prenatal, intrapartum and neonatal period. India is still facing a high (25.4/1000) perinatal mortality rate despite moderns obstetrics and neonatal services (target is 12/1000). Primary reason for this poor performance is failure to identify in time the mother and foetus at risk. Perinatal outcome can be changed remarkably by early detection and appropriate management. Hence this study aims to detect high risk cesarean cases with life threatening obstetric maternal and fetal complication by simple risk scoring analysis for better pregnancy outcome especially in low resource setting.

\section{METHODS}

This prospective analytical study was conducted in Department of Obstetrics and Gynaecology at JK Hospital and LN Medical college, Bhopal. 120 pregnant women primipara, multipara with term pregnancy and age group of 18-35Years, admitted for delivery during June 2017 till May 2018 and planed for emergency cesarean section irrespective of their booked or unbooked status were included in the study. Normal deliveries were excluded from the study, Detailed history (reproductive, medical or surgical associated condition, present pregnancy) was taken and thorough physical and obstetric examination was done with necessary investigations in each patient to determine the risk factors (Table 1). Observations were documented in modified risk scoring system based on predesigned and pretested risk factors validated from previous studies. independent risk score was added to give a total score to classify pregnancy in to low risk $(<3)$ intermediate risk $(3-6)$ and high risk (>6) group.

On multivariate analysis maternal outcome in terms of HDU admission and morbidity as postpartum hemorrhage, multi organ failure, convulsion, sepsis, mortality were observed and fetal outcome - birth weight <2500gms, APGAR score, birth asphyxia, perinatal mortality, intrauterine death, still birth and early neonatal death were recorded (Table 2). Observations of both the groups were compared with the respective scores for statistical analysis by Chi square test as test of significance and calculated $\mathrm{P}$ value of $<0.5$ was taken as statistically significant.

Table 1: Obstetric risk category and subsequent risk score wise distribution of study subjects.

\begin{tabular}{|lll|}
\hline Obstetric risk category & Score & Frequency \\
\hline Pre eclampsia/eclampsia & 3 & 21 \\
\hline Anemia /Malnutrition & 2 & 33 \\
\hline Medical disorder & 2 & 5 \\
\hline Previous cesarean & 3 & 20 \\
\hline Prom & 2 & 9 \\
\hline prolonged labour & 3 & 6 \\
\hline Post maturity & 2 & 3 \\
\hline Previous still birth/NND & 3 & 1 \\
\hline APH/Placenta previa & 2 & 4 \\
\hline IUGR & 2 & 19 \\
\hline Oligohydramnious & 2 & 17 \\
\hline Malpresentation & 1 & 3 \\
\hline multiple pregnancy & 3 & 2 \\
\hline Age>34 <18 & 3 & 23 \\
\hline Parity>3, Primipara & 3 & $5+52$ \\
\hline h/o abortion /infertility t/t & 2 & 4 \\
\hline Meconium stained liquor MAS & 2 & 1 \\
\hline PPH/MRP & 1 & 1 \\
\hline bleeding before 20 weeks & 2 & 3 \\
\hline Ho Gynec. surgery & 1 & 2 \\
\hline Polyhydramnious & 2 & 5 \\
\hline
\end{tabular}

Table 2: Description of pregnancy outcome of study subjects.

\begin{tabular}{|ll|}
\hline Pregnancy outcome & Frequency \\
\hline Low birth weight & 30 \\
\hline Still birth & 1 \\
\hline Perinatal mortality & 1 \\
\hline Low APGAR 4-6 & 22 \\
\hline Apgar $<4$ & 16 \\
\hline Apgar $>6$ & 82 \\
\hline
\end{tabular}

\section{RESULTS}

In this prospective analytical study, a total of 120 at risk pregnant women for emergency cesarean were grouped as HRP $25 \%$ (30), 16.6\% (20) intermediate risk and 70 $(58.3 \%)$ as LRP on the basis of cumulative score.

Table 3: Effects of obstetric risk level on pregnancy outcome.

\begin{tabular}{|c|c|c|c|c|}
\hline \multirow{2}{*}{$\begin{array}{l}\text { Obstetric } \\
\text { risk level }\end{array}$} & \multicolumn{2}{|c|}{ Pregnancy outcome } & \multirow{2}{*}{ Total } & \multirow{2}{*}{$\begin{array}{l}\text { Significance } \\
\text { level }\end{array}$} \\
\hline & Normal & Abnormal & & \\
\hline High risk & 3 & 27 & 30 & \multirow{4}{*}{$\begin{array}{l}P \text { value }= \\
<0.001\end{array}$} \\
\hline $\begin{array}{l}\text { Moderate } \\
\text { risk }\end{array}$ & 14 & 6 & 20 & \\
\hline Low risk & 65 & 5 & 70 & \\
\hline Total & 82 & 38 & 120 & \\
\hline
\end{tabular}


$15.8 \%$ of the mothers were in the age group of $18-34$ years with $68.42 \%$ of abnormal outcome, (Table 3) $52.5 \%$ mothers were primipara with morbidity of $44.44 \%$ while remaining $47.5 \%$ of mothers were para three with the perinatal morbidity of $17.4 \%$ (Table 4 ).

Table 4: Effects of parity on pregnancy outcome.

\begin{tabular}{|c|c|c|c|c|}
\hline \multirow{2}{*}{ Parity } & \multicolumn{2}{|c|}{ Pregnancy outcome } & \multirow{2}{*}{ Total } & \multirow{2}{*}{$\begin{array}{l}\text { Significance } \\
\text { level }\end{array}$} \\
\hline & Normal & Abnormal & & \\
\hline Primipara & 35 & 28 & 63 & \multirow{3}{*}{$\begin{array}{l}\mathrm{P} \text { value }= \\
0.002\end{array}$} \\
\hline Multipara & 47 & 10 & $52+5$ & \\
\hline Total & 82 & 38 & 120 & \\
\hline
\end{tabular}

Chi-Square value $=10.0, \mathrm{df}=1$

$40 \%$ babies in the HR group and $15 \%$ babies in the moderate group were severe depression with Apgar score below 4 and admitted in the NICU while only $1.4 \%$ babies of low risk needed admission. APGAR score a good determinant of intrapartum hypoxia shows that complications could be prevented if identified in time and babies delivered in optimum conditions before they worsened to irreversible stage. perinatal outcome remains grim in low APGAR with severe hypoxia in fetus despite good resuscitative measures undertaken in a newborn, one intra partum demise in case of eclampsia and another baby died due to RDS of high risk group (Table 7).

Present study observed anemia with malnutrition $27.5 \%$ is one of the commonest complications of pregnancy and had a harmful effect on reproductive outcome as it causes preterm deliveries or intrauterine deaths, poor maternal weight gains intra- uterine growth retardation, preterm labor and accounted for $47 \%$ of the perinatal morbidity.

$2^{\text {nd }}$ commonest complication was PIH (Pregnancy induced hypertension) $17.5 \%$ and accounting for high perinatal mortality and morbidity. It is gathered from this study that incidence of medical disease (4.1\%) in pregnancy has a reasonably good perinatal outcome as these diseases are detected and managed early in pregnancy. Mothers with twin pregnancy have greater perinatal morbidity as it carries a very high risk to the fetus primarily due to low birth weight with prematurity and birth trauma, asphyxia, and operative intervention. Abnormal FHR as determined clinically as well as by electronic fetal monitor during labor portray intrauterine fetal hypoxia which is one of the major contributing factors for poor perinatal outcome.

In present study abnormal FHR accounted for one perinatal death. Induction of labor directly does not affect perinatal outcome except due to iatrogenic preterm delivery in some cases, but indirectly it may affect outcome for the indication it was done. premature rupture of membrane is one of the important determinants of perinatal morbidity $(7.5 \%)$ as it is associated with increased incidence of perinatal infections and deaths. Presence of meconium was seen in 5\% cases in present study group with a resultant morbidity of $20 \%$ (Table 5).
Higher incidence $(46.6 \%)$ of low birth weight babies was seen in the mothers who had more than one risk factors (Table 6).

Table 5: Effects of maternal age on pregnancy outcome.

\begin{tabular}{|c|c|c|c|c|}
\hline \multirow{2}{*}{$\begin{array}{l}\text { Maternal } \\
\text { Age }\end{array}$} & \multicolumn{2}{|c|}{ Pregnancy outcome } & \multirow{2}{*}{ Total } & \multirow{2}{*}{$\begin{array}{l}\text { Significance } \\
\text { level }\end{array}$} \\
\hline & Normal & Abnormal & & \\
\hline$\leq 34$ & 76 & 25 & 101 & \multirow{3}{*}{$\begin{array}{l}\mathrm{P} \text { value }= \\
<0.001\end{array}$} \\
\hline$>34 /<18$ & 6 & 13 & 19 & \\
\hline Total & 82 & 38 & 120 & \\
\hline
\end{tabular}

Chi-Square value $=14.1, \mathrm{df}=1$

High risk category of $(25 \%)$ had highest perinatal mortality of 2 and maternal mortality of one with total 3 admission in high dependency unit (HDU). HRP had poor perinatal outcome as compare to low risk cases.

Table 6: Effects of obstetric risk level on birth weight of baby.

\begin{tabular}{|c|c|c|c|c|}
\hline \multirow{2}{*}{$\begin{array}{l}\text { Obstetric } \\
\text { risk level }\end{array}$} & \multicolumn{2}{|c|}{ Birth weight } & \multirow{2}{*}{ Total } & \multirow{2}{*}{$\begin{array}{l}\text { Significance } \\
\text { level }\end{array}$} \\
\hline & Normal & Low & & \\
\hline High risk & 16 & 14 & 30 & \multirow{4}{*}{$\begin{array}{l}\mathrm{P} \text { value }= \\
<0.001\end{array}$} \\
\hline $\begin{array}{l}\text { Moderate } \\
\text { risk }\end{array}$ & 11 & 9 & 20 & \\
\hline Low risk & 63 & 7 & 70 & \\
\hline Total & 90 & 30 & 120 & \\
\hline
\end{tabular}

Chi-Square value $=20.2, \mathrm{df}=2$

Maternal outcome was analysed by HDU admission 3\% mothers were admitted in HDU with eclampsia and multi organ failure with one maternal mortality due to HELLP syndrome other recovered well, while one patient left against medical advice due to high expenses. The difference in three categories was statistically significant. Present study based on authors institutional disposition. Which gathers mostly rural population with high referral rate, so study cannot be generalized or compared with any other study therefore we would like to conduct subsequent study based on appropriate sampling technique and proper sample size.

Table 7: Effects of obstetric risk level on APGAR score of babies.

\begin{tabular}{|c|c|c|c|c|c|}
\hline \multirow{2}{*}{$\begin{array}{l}\text { Obstetric } \\
\text { risk level }\end{array}$} & \multicolumn{3}{|c|}{ APGAR Score } & \multirow{2}{*}{ Total } & \multirow{2}{*}{$\begin{array}{l}\text { Significance } \\
\text { level }\end{array}$} \\
\hline & $<4$ & 4 to 6 & $>6$ & & \\
\hline High risk & 12 & 15 & 3 & 30 & \multirow{4}{*}{$\begin{array}{l}P \text { value }= \\
<0.001\end{array}$} \\
\hline $\begin{array}{l}\text { Moderate } \\
\text { risk }\end{array}$ & 3 & 3 & 14 & 20 & \\
\hline Low risk & 1 & 4 & 65 & 70 & \\
\hline Total & 16 & 22 & 82 & 120 & \\
\hline
\end{tabular}

\section{DISCUSSION}

Any pregnancy can develop life threatening complications at any time with or without any warning. Caesarean section is the most common obstetric surgery performed to save mother and baby in emergency is now 
safer due to advances in techniques, availability of blood and better antibiotics, still the maternal and neonatal mortality and morbidity is much higher in developing countries, due to inadequate health facilities, so concomitant decrease in perinatal mortality has not been substantiated by an increase in the rate of CS in developing countries, Majority of high risk patient seen in daily practice are women who in course of otherwise normal pregnancy develop unexpected severe complications, these patient with unexpected complications require difficult management decision and when a poor fetal or maternal outcome occurs the obstetrician is faced with the possibility of medicolegal problems. Papoutsis also developed the cesarean section risk assessment tool to comprehend accurately the predication of pregnancy outcome and found it helpful in decision making in HRP cases. ${ }^{3}$

In present study it is evident that the maximum number of babies were less than $2.5 \mathrm{~kg}$ in both the high risk and moderate risk groups, Rijal $\mathrm{P}$, in his study found that out of total $9.4 \%$ newborns were weighing less than $2.50 \mathrm{~kg}$ and mean birth weight $2.69 \mathrm{~kg} .{ }^{4}$ This study suggests that interplaying of several factors like age of mother, weight gain during pregnancy, short stature, low body mass index and hyperemesis gravidarum lead to LBW babies, and became strongest predictors in this study. Near similar observations were reported by Samiya M. ${ }^{5}$

Low birth weight, which simply signifies that the baby is born malnourished, is a formidable challenge for India. In our study we observed that the incidence of perinatal morbidity was more in high risk group, low birth weight and prematurity was the most common perinatal morbidity $(25 \%)$ followed by respiratory distress $(9 \%)$ in this group. A prospective study conducted by Le Fevre M et al in their obstetric risk assessment in rural practice forty-seven pregnancies $(8.3 \%)$ had an adverse outcome in terms of newborn transferred to level 2 or level 3 nursery. ${ }^{6}$ There was a clear relationship between risk score and probability of adverse outcome where low risk group mothers had 50\% lower incidence of high risk neonates $(41.3 \%)$ as compared to high risk group mothers who had $84.4 \%$ of high risk neonates as in present study.

HRP is multifactorial so more than one direct or indirect factor contribute for mortality and morbidity as intrapartum events like abnormal progress of labor, meconium stained liquor, fetal heart rate variation, mal presentation, induced labor, IUGR. It indicates that advanced perinatal care system emphasizes on routine screening services for risk factors associated with adverse pregnancy. The rural units lack skilled manpower, equipment's and other facilities to handle obstetric emergency. Poor referral system, low socioeconomic status, illiteracy, and inadequate antenatal care, inadequate transport facilities to apex hospital-all this leads to increased risk of maternal and perinatal complications. obstructed labour (34\%) was the commonest indication in emergency and previous caesarean delivery (36\%) being the commonest in elective group. The live birth was $88 \%$ in Emergency group as against $100 \%$ live births in Elective group. Perinatal mortality from emergency Caesarean sections accounted for $12 \%$, with severe birth asphyxia responsible for most perinatal deaths and maternal mortality. ${ }^{7}$

Khunpradit shows the risk of cesarean delivery due to CPD may be forecasted by a simple scoring scheme using five predictors: maternal age, height, parity, pregnancy weight gain and symphysis-fundal height and scores ranged from 0 up to 3.5 that correctly identified women with low, moderate and high risk. ${ }^{8}$ Study advocates scheme may be applicable to physicians and midwives for identifying high-risk pregnant women in order to take appropriate action as similar to our study.

Sodsee S, study proposes modified nearest neighbor analysis which is called CPD-NN algorithm to approximate risk about CS due to CPD. ${ }^{9}$ Cesarean section is the first major operation to prevent child birth complications from high risk pregnancy, maternal mortality and childbirth complications are major problem of delivery in rural area of many developing countries. In information era, it would be beneficial if the risk of delivery could be informed or recommended to patients at earlier sign. As well as, physicians could draw approximate decision before it occurred.

In present study one maternal death was due to multi organ failure due to eclampsia, study of Khandale SN, also confirms maternal mortality due to hypertensive disorder of pregnancy $28.19 \% .^{10}$ this address the need for HDU set up in all obstetric catering center. This study also observed the type of delay to the tertiary center is an important contributory factor for MMR. that's why this simple, economical and handy scoring system should be applied in all cases admitted for delivery to document and predict the outcome well ahead of time. ${ }^{11}$

\section{CONCLUSION}

High maternal and perinatal morbidity and mortality is because of failure to identify the risk in time, pregnancy outcome can be changed by early detection of complication. So, this simple, costless, handy scoring system not only help in documenting high risk pregnancy in this medicolegal era but also elaborate prognosis thus help in treating less demanding cases at PHC and funnel serious cases to specialist in well-equipped medical center thus have a positive impact in lowering the maternal and perinatal mortality and morbidity from base level only.

\section{Funding: No funding sources Conflict of interest: None declared \\ Ethical approval: The study was approved by the Institutional Ethics Committee}




\section{REFERENCES}

1. De A, De A, Fatima A, Sathian B, Asgil T, Finnegan R, Sharma JS. Prenatal risk score and pregnancy outcome: a study in Nepal. Res J Pharmaceut Bio Chem Sci. 2015 May 1;6(3):807-12

2. Honest H, Bachmann LM, Sundaram R, Gupta JK, Kleijnen J, Khan KS. The accuracy of risk scores in predicting preterm birth-a systematic review. J Obstet Gynaecol. 2004 Jun 1;24(4):343-59.

3. Papoutsis D, Antonakou A, Gornall A, Tzavara C, Mohajer M. The SaTH risk-assessment tool for the prediction of emergency cesarean section in women having induction of labor for all indications: a largecohort based study. Arc Gynecol Obstetr. 2017 Jan 1;295(1):59-66.

4. Rijal P. Identification of risk factors for cesarean delivery following induction of labour. J Nepal Health Res Council. 2014;12(27):73-7.

5. Sowmya M, Dutta I. Comparative study of neonatal outcome in ceasarean section done in referred cases vs elective ceasarean delivery in a rural medical college hospital. J Evol Med Dental Sci. 2014 Nov 24;3(64):13993-9.

6. LeFevre M, Williamson HA, Hector JM. Obstetric risk assessment in rural practice. J Fam Pract. 1989 Jun;28(6):691-5.

7. Vogel JP, Souza JP, Mori R, Morisaki N, Lumbiganon $\mathrm{P}$, Laopaiboon $\mathrm{M}$, et al. Maternal complications and perinatal mortality: findings of the World Health Organization multicountry survey on maternal and newborn health. BJOG: Int J Obstetr Gynaecol. 2014 Mar;121:76-88.

8. Khunpradit S, Patumanond J, Tawichasri C. Development of risk scoring scheme for prediction of cesarean delivery due to cephalopelvic disproportion in Lamphun Hospital, Thailand. J Obstetr Gynaecol Res. 2007 Aug 1;33(4):445-51.

9. Sodsee S. Predicting caesarean section by applying nearest neighbor analysis. Procedia Computer Scince. 2014 Jan 1;31:5-14.

10. Khandale SN, Kedar K. Analysis of maternal mortality: a retrospective study at tertiary care centre. Int J Reprod Contracept Obstetr Gynecol. 2017 Mar 30;6(4):1610-3.

11. James DK, Steer PJ, Weiner CP, Gonik B. High risk pregnancy E-Book: Management options-expert consult. Elsevier Health Sciences; 2010 Dec 3.

Cite this article as: Jain SB, Tripathi R. Antipartum surveillance by scoring system for pregnancy outcome of cesarean section in a tertiary referral centre of Madhya Pradesh, India. Int J Reprod Contracept Obstet Gynecol 2018;7:3558-62. 